\title{
Communication
}

\section{Intermediate dipolar distances from spin labels}

\author{
Derek Marsh \\ Max-Planck-Institut für biophysikalische Chemie, 37070 Göttingen, Germany
}

\section{A R T I C L E I N F O}

\section{Article history:}

Received 30 August 2013

Revised 1 November 2013

Available online 16 November 2013

\section{Keywords:}

Inter-spin distances

Dipolar interactions

Second moment

Absolute first moment

Distance distribution

Spin label

\begin{abstract}
A B S T R A C T
Methods for determining inter-spin distances between nitroxide spin labels from dipolar couplings in the intermediate range $\left(r_{12} \approx 1.1-2 \mathrm{~nm}\right)$ by CW-EPR are addressed. For nitroxide powder patterns, the assumption of unlike spins is a better approximation than assuming strong coupling between like spins. Methods that determine the average splitting in dipolar deconvolutions yield the mean value $\left\langle 1 / r_{12}^{3}\right\rangle$, and those that determine the dipolar contribution to the spectral second moment correspondingly yield $\left\langle 1 / r_{12}^{6}\right\rangle$. To relate these ensemble averages to the mean inter-spin distance $\left\langle r_{12}\right\rangle$ requires knowledge of the distribution in $r_{12}$. Values of the inverse roots $r_{e f f, n}=\left\langle 1 / r_{12}^{n}\right\rangle^{-1 / n}$ always lie below $\left\langle r_{12}\right\rangle$. Consistent comparisons of literature data on double-labelled $\alpha$-helical peptides support this view. Evaluations of $r_{e f f, n}$ for Gaussian distributions in $r_{12}$ yield calibrations to determine $\left\langle r_{12}\right\rangle$ for a given distribution width, $\sigma_{r_{12}}$.
\end{abstract}

(c) 2013 Elsevier Inc. All rights reserved.

\section{Introduction}

Useful information on inter-spin distances, $r_{12}$, can be obtained from standard CW-EPR spectra in the intermediate regime $\left(r_{12} \approx 1.1-2 \mathrm{~nm}\right)$, when the dipolar coupling is less than the width of the powder spectrum of an isolated spin label [1-4]. This is particularly valuable in the field of site-directed spin labeling where, however, a distribution of distances arises from the flexibility of the spin-label attachment. Under these circumstances, the nitroxide may have a statistical distribution of orientations for each orientation of the inter-spin vector, and analysis by spectral convolution [5] or deconvolution [6] is possible. Convolution is performed by assuming a Gaussian distribution of distances [5,7], whereas deconvolution yields a dipolar powder line shape that may be fitted to deduce the distance distribution [8]. In cases where the deconvoluted powder line shape is relatively featureless, a simplified analysis has been introduced that is based on the average splitting [6,9]. A further simplified approach is to determine the dipolar contribution to the spectral second moment $[10,11]$, for which an analytical solution is available in the manybody case [12].

The assumption often made, or implied, is that the different methods of analysis should yield the same mean distance. This is not true, however, if there is a distribution of distances. As will be seen below, the average splitting is related to the mean value of $1 / r_{12}^{3}$, and the second moment to that of $1 / r_{12}^{6}$, and not directly to the mean of $r_{12}$. A further point is the lack of consistency between different authors as to whether the assumption of like or

E-mail address: dmarsh@gwdg.de of unlike spins (i.e., strong or weak coupling) is appropriate in this regime. The purpose of this note is to address these problems and offer suggestions for solution.

\section{Magnetic dipole-dipole interaction for like and unlike spins}

To an acceptable degree of approximation, the spin Hamiltonian for the magnetic dipole-dipole interaction between two spins $s_{1}$ and $s_{2}$ is restricted to the secular and pseudo-secular terms:

$\widehat{\mathscr{H}}_{d d}=\frac{g^{2} \beta_{e}^{2}}{r_{12}^{3}}\left(1-3 \cos ^{2} \theta\right)\left[s_{1 z} s_{2 z}-\frac{1}{4}\left(s_{1+} s_{2-}+s_{1-} s_{2+}\right)\right]$

where $r_{12}$ is the inter-spin distance, $\theta$ is the inclination of the interspin vector to the static magnetic field, $g$ is the $g$-value of each spin (the small $g$-value anisotropy can be neglected for nitroxides), and $\beta_{e}$ is the Bohr magneton. For like spins, i.e., those for which the dipolar coupling (and/or exchange) is considerably larger than the difference in their resonance frequency, the pseudosecular terms in Eq. (1) couple the quasi-degenerate individual spins $s_{1}=\frac{1}{2}$ and $s_{2}=\frac{1}{2}$ to a triplet or singlet state for which the total spin is: $S=s_{1}+s_{2}=1$ or 0 , respectively. EPR transitions occur only within the triplet, according to the selection rules $\Delta S=0$ and $\Delta M_{S}= \pm 1$ for magnetic dipole transitions. The spin-spin splitting of the resonance magnetic field in the EPR spectrum for a pair of like spin labels is then given by:

$\pm \Delta H_{d d}(\theta)=\frac{3}{4} \frac{g \beta_{e}}{r_{12}^{3}}\left(1-3 \cos ^{2} \theta\right)$

and exchange does not enter. For unlike spins, i.e., those for which the dipolar coupling (and exchange) is smaller than their difference 
in resonance frequency, the pseudosecular terms in Eq. (1) remain off-diagonal, contribute only in second order, and thus may be neglected. The spin-spin splitting of the resonance magnetic field in the EPR spectrum of an unlike spin-label pair is then given by:

$\pm \Delta H_{d d}(\theta)=\frac{1}{2} \frac{g \beta_{e}}{r_{12}^{3}}\left(1-3 \cos ^{2} \theta\right)$

if exchange can be neglected. This is two-thirds of that for a like spin-label pair (cf. Eq. (2)). Clearly, when comparing different methods of distance measurement on the same system, a consistent assumption of either like or unlike spins is essential.

Electron spins are unlike if they are in different hyperfine states, or have different orientations to the magnetic field, and the dipolar coupling is less than the resulting difference in resonance frequency. Differences in orientation arise for asymmetrical rigid biradicals, or more commonly from the innate flexibility of the attachment of the two spin labels. The distinction between like and unlike spins in a $9-\mathrm{GHz}$ powder spectrum is illustrated by Fig. 1. Grey and light-grey shaded areas correspond to dipolar splittings of 5 and 20 gauss, respectively, which correspond to separations between unlike spins of $r_{12}=1.55$ and $0.98 \mathrm{~nm}$ (1.77 and $1.12 \mathrm{~nm}$ for like spins). The dipolar splitting of 5 gauss does not span even the narrowest, central $m_{I}=0$ hyperfine manifold of the nitroxide powder spectrum. For this we need $2 \Delta H_{d d} \approx 20$ gauss. Then the $m_{I}=0$ spins are like spins, but the $m_{I}= \pm 1$ spins are not because these two manifolds spread over much wider ranges of spectral anisotropy. Therefore only one ninth of the spin pairs consist of like spins, and the remaining spin pairs are unlike. This situation becomes more extreme on going to higher EPR frequencies. Thus, whenever the dipolar coupling is considerably smaller than the overall extent of the single-nitroxide powder pattern, which includes the intermediate distance range, the better approximation is to consider all spins as being unlike.

It should be noted that Heisenberg exchange contributes to spin-spin broadening under all circumstances for unlike spins. Exchange interactions then can be neglected only if they are numerically insignificant compared with the magnetic dipole-dipole interaction. Sometimes it is implied that exchange does not contribute to the second moment, but this is true only for indistinguishable, i.e., like, spins. Exchange decays more rapidly with distance than does the dipolar interaction. Spectral simulation shows that isotropic exchange is negligible compared with dipolar interactions for unlike nitroxides with separations in the intermediate range: $r_{12}=1.28-1.56 \mathrm{~nm}$ [13].

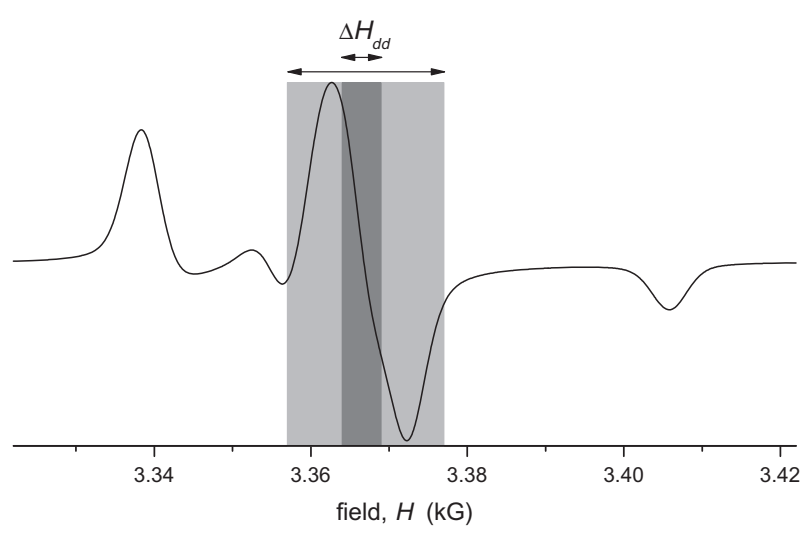

Fig. 1. Like and unlike spins. Like and unlike nitroxide spins in a 9.4-GHz powder spectrum. The grey-shaded area corresponds to a dipolar powder pattern of width $\Delta H_{d d}=5$ gauss, and the light-grey area to $\Delta H_{d d}=20$ gauss.

\section{Dipolar powder spectra and distance distributions}

For a randomly oriented sample, a dipolar powder spectrum is obtained by summing Eq. (2) or (3) over all angles $\theta$. With a fixed separation between spins, this yields the so-called Pake pattern [14] that is characterized by sharp discontinuities at $\pm \Delta H_{d d}\left(90^{\circ}\right)$ and outer shoulders at $\pm \Delta H_{d d}\left(0^{\circ}\right)$. The profile of the Pake pattern is given by the identity: $I\left(\Delta H_{d d}\right) \cdot d \Delta H_{d d}=\sin \theta \cdot d \theta$, which corresponds to the angular distribution for axial symmetry. This results in a normalized powder envelope that is given by:

$I\left(\Delta H_{d d}\right)=\frac{1}{2 \sqrt{3 D_{d d}}} \frac{1}{\sqrt{D_{d d}-\Delta H_{d d}}}$

where $D_{d d}=\frac{3}{4} g \beta_{e} / r_{12}^{3}$ for like spins, and $D_{d d}=\frac{1}{2} g \beta_{e} / r_{12}^{3}$ for unlike spins.

Frequently, spin-label attachments are not rigid, which results in an approximately Gaussian distribution of inter-spin distances:

$p\left(r_{12}\right)=\frac{1}{\sigma_{r_{12}} \sqrt{2 \pi}} \exp \left(-\frac{\left(r_{12}-\left\langle r_{12}\right\rangle\right)^{2}}{2 \sigma_{r_{12}}^{2}}\right)$

where $\left\langle r_{12}\right\rangle$ and $\sigma_{r_{12}}$ are the mean and standard deviation, respectively. The full dipolar powder pattern is then a sum of normalized Pake doublets that are weighted by $p\left(r_{12}\right)$ for each value of $r_{12}$, as is illustrated in Fig. 2. The splitting of the apparent doublet peaks in the resulting spectral envelope is less than that corresponding to the mean inter-spin distance, by an amount that increases with increasing distribution width. Also, the outer shoulders that are characteristic of a single Pake doublet are not resolved in the overall envelope. Although the range displayed in Fig. 2 covers most of that over which the line height is appreciable, the spectral envelope extends out to splittings four times those shown. This points to potential difficulty in determining the full extent of the shorter side of the distribution function, which in any case is limited by the experimental sweep width. Nonetheless, fitting from a library of Pake doublets by convolution or deconvolution, with or without assuming a Gaussian distribution, should be sufficient to determine the true mean distance and its distribution width. It is not true, however, that single metrics such as the average splitting or the second moment alone can yield true mean distances, without knowledge of the distribution width.

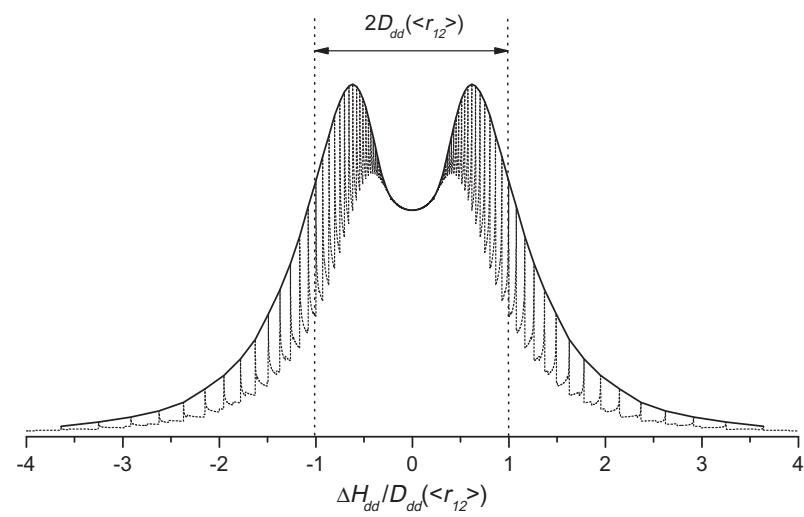

Fig. 2. Distribution of dipolar powder patterns. Dipolar powder pattern for a Gaussian distribution of inter-spin distances $r_{12}$, with standard deviation: $\sigma_{r_{12}}=0.33 \times\left\langle r_{12}\right\rangle$. Dashed line is a superposition of dipolar envelopes for each inter-spin distance. The peaks of the envelope corresponding to the mean separation are at $\pm D_{d d}\left(\left\langle r_{12}\right\rangle\right)$ - see vertical dotted lines. For unlike spins: $D_{d d}\left(r_{12}\right) \equiv \frac{1}{2} g \beta_{e} / r_{12}^{3}$. 


\section{Average dipolar splittings and second moments}

Fourier deconvolution can be used to extract dipolar absorption powder patterns from experimental spectra of interacting spin labels as described in Refs. [6,8]. The average splitting is a useful means to analyse dipolar deconvolutions that lack the features characteristic of dipolar Pake patterns [6], or are forced into Gaussian shapes by fitting in Fourier space [9]. Formally, the mean splitting is the absolute first moment (or twice the one-sided first moment), which for a single Pake powder pattern is given by:

$\left\langle 2\left|\Delta H_{d d}\right|\right\rangle=\int_{-2 D_{d d}}^{D_{d d}} 2\left|\Delta H_{d d}\right| \cdot I\left(\Delta H_{d d}\right) d \Delta H_{d d}=\frac{4}{3 \sqrt{3}} 2 D_{d d}$

where $I\left(\Delta H_{d d}\right)$ is the normalized envelope of half a Pake doublet, and $2 D_{d d}=g \beta_{e} / r_{12}^{3}$ for unlike spins (see upper panel of Fig. 3 ). With a distribution of distances $r_{12}$, the contributions of the different Pake patterns to the average splitting are additive. From Eq. (5), the experimental average splitting then gives the weighted average of $D_{d d}$, i.e., the mean value $\left\langle 1 / r_{12}^{3}\right\rangle$.

Dipolar contributions to the second moment of EPR absorption spectra from systems of interacting spin labels can be determined from experimental spectra of the doubly and single labeled systems as indicated in the lower panel of Fig. 3. In practice, the
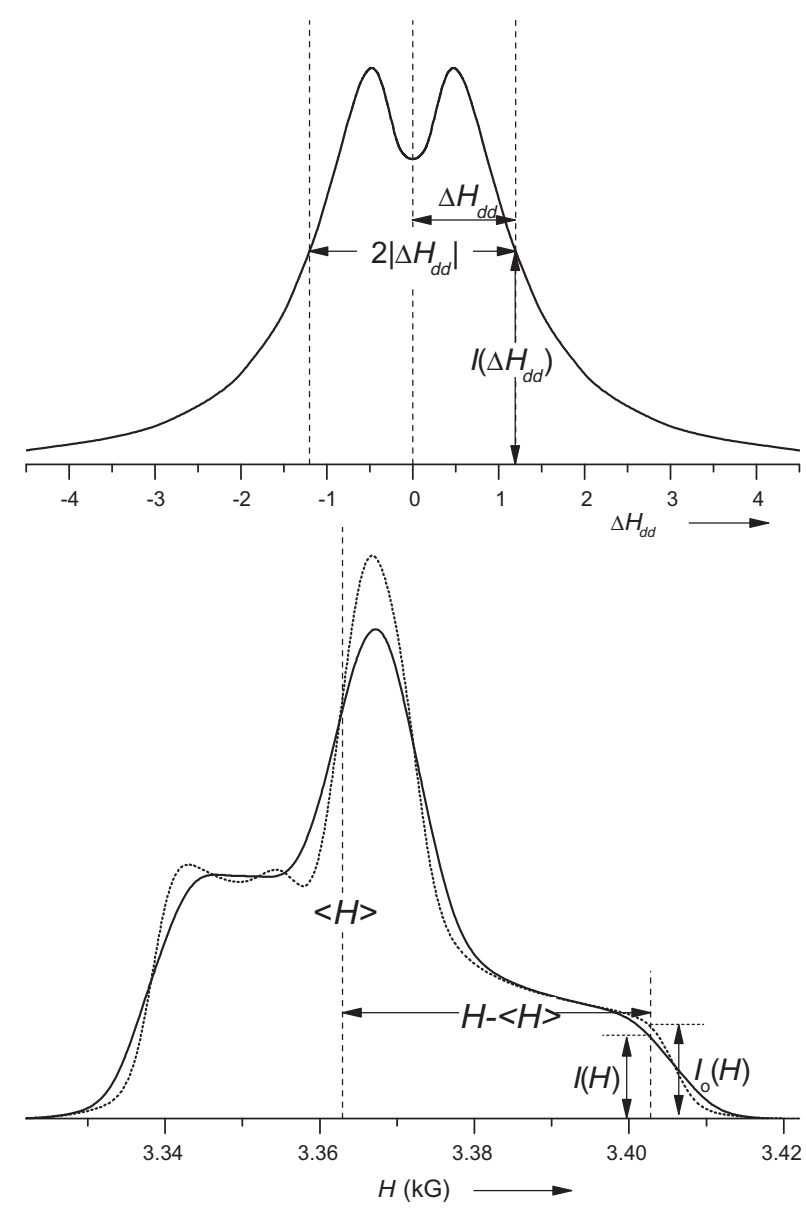

Fig. 3. Determination of mean dipolar splitting and second moment. Upper panel: mean dipolar splitting (absolute first moment), $\left\langle 2\left|\Delta H_{d d}\right|\right\rangle$, for a deconvoluted absorption dipolar powder pattern is given by Eq. (6) with the definitions in the figure. Lower panel: dipolar second moment $\left\langle\Delta H_{d d}^{2}\right\rangle=\left\langle H^{2}\right\rangle-\left\langle H_{o}^{2}\right\rangle$, where $\left\langle H^{2}\right\rangle$ is the second moment in the presence of dipolar broadening (solid-line absorption spectrum), and $\left\langle H_{0}^{2}\right\rangle$ that in the absence of spin-spin broadening (dotted-line absorption spectrum). The second moments are calculated about the mean field position $\langle H\rangle$ (see text). second moment $\left\langle(\Delta H)^{2}\right\rangle$ is best calculated about the mean resonance position, $\langle H\rangle$, because this corresponds to the isotropic $g$-value [15] and therefore compensates for any differences in field/frequency of the different samples. Thus $\left\langle(\Delta H)^{2}\right\rangle=$ $\left\langle(H-\langle H\rangle)^{2}\right\rangle \equiv \int(H-\langle H\rangle)^{2} I(H) \cdot d H / \int I(H) \cdot d H$, where $\langle H\rangle$ is the first moment.

Similarly to the mean splitting, the dipolar second moment for a single Pake powder pattern is given by:

$\left\langle\Delta H_{d d}^{2}\right\rangle=\int_{-2 D_{d d}}^{D_{d d}}\left(\Delta H_{d d}\right)^{2} \cdot I\left(\Delta H_{d d}\right) d \Delta H_{d d}=\frac{4}{5}\left(D_{d d}\right)^{2}$

For an assembly of spins, the corresponding general result for the dipolar second moment in a powder sample of unlike spins is well known [12]:

$\left\langle\Delta H_{d d}^{2}\right\rangle=\frac{4}{15} s^{\prime}\left(s^{\prime}+1\right) g^{2} \beta_{e}^{2} \sum_{k} 1 / r_{1 k}^{6}$

where the summation is over all unlike spins $k$, for each of which $s^{\prime}=1 / 2$. With a distribution of inter-spin distances, the second moment therefore gives the mean value $\left\langle 1 / r_{12}^{6}\right\rangle$. Both these mean values, viz., $\left\langle 1 / r_{12}^{n}\right\rangle$, depend not only on the mean inter-spin distance, but also on the width of the distance distribution.

\section{Mean values of $1 / r_{12}^{n}$ and effective distances}

The necessary mean values are determined by integration over the probability distribution, $p\left(r_{12}\right)$ :

$\left\langle\frac{1}{r_{12}^{n}}\right\rangle=\int_{r_{\min }}^{\infty} \frac{1}{r_{12}^{n}} p\left(r_{12}\right) \cdot d r_{12}$

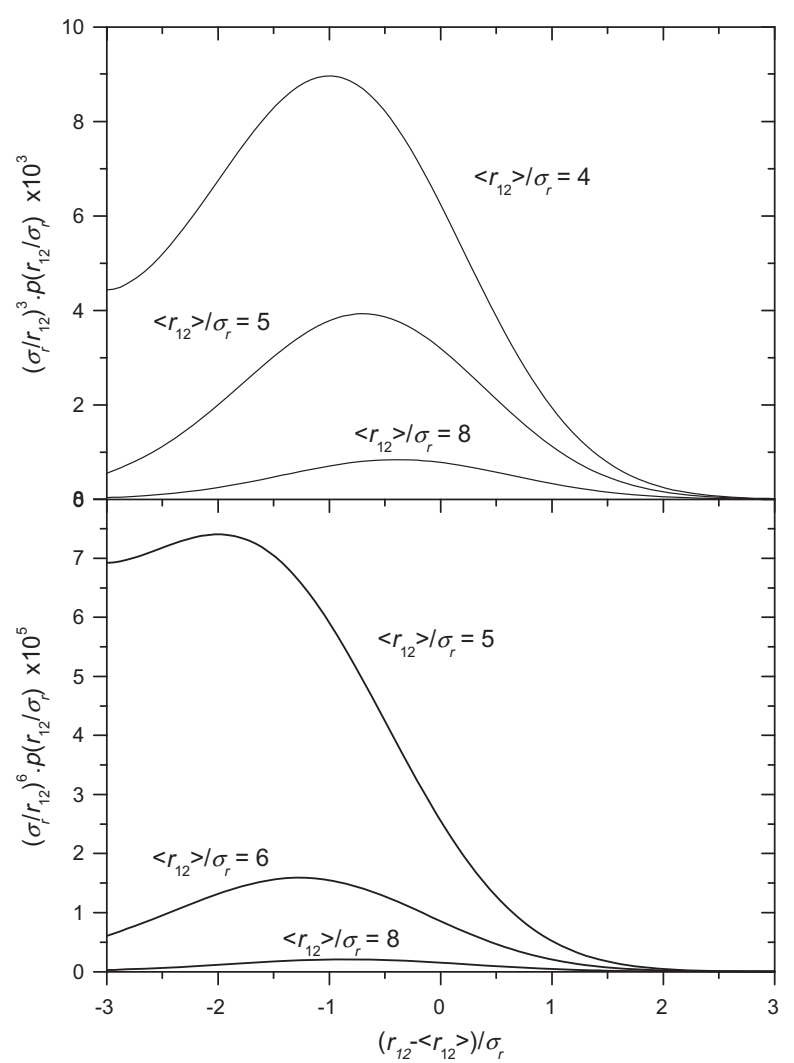

Fig. 4. Distribution of $1 / r_{12}^{3}$ and $1 / r_{12}^{6}$. Dependence of the weighted values of $1 / r_{12}^{3}$ (upper panel) and of $1 / r_{12}^{6}$ (lower panel) on inter-spin distance $r_{12}$, for a Gaussian distribution $p\left(r_{12}\right)$ of $r_{12}$ with standard deviation $\sigma_{r_{12}}$ and mean value $\left\langle r_{12}\right\rangle$. All distances are normalized to the value of $\sigma_{r_{12}}$. 
where $n=3$ or 6 , and $r_{\min }$ is some suitable cut-off that is chosen to prevent the integral from diverging. Fig. 4 shows values of $1 / r_{12}^{3}$ (top panel) and of $1 / r_{12}^{6}$ (bottom panel) that are weighted by Gaussian distance distributions (i.e., Eq. (4)) with different widths. At values of $r_{12}-\left\langle r_{12}\right\rangle$ more negative than those shown in the figure, the ordinate begins to diverge because of the inverse power dependence on $r_{12}$. For the largest distribution width shown $\left(\sigma_{r_{12}}=0.25\left\langle r_{12}\right\rangle\right.$ in the top panel, and $\sigma_{r_{12}}=0.2\left\langle r_{12}\right\rangle$ in the bottom panel), this is already evident from the incipient minima at the far left. The numerical instability is more extreme when evaluating $\left\langle 1 / r_{12}^{6}\right\rangle$ than it is for $\left\langle 1 / r_{12}^{3}\right\rangle$.

Restricting the integration in Eq. (9) to the range displayed in Fig. 4, the dependences of $r_{\text {eff. } n}=\left\langle 1 / r_{12}^{n}\right\rangle^{-1 / n}$ on the distribution width are shown for $n=3$ and 6 in Fig. 5. The effective values are consistently lower than the true mean, to an extent that depends on the distribution width and is more pronounced for $n=6$ than for $n=3$. Even for a relatively narrow distribution width $\sigma_{r_{12}}=0.1\left\langle r_{12}\right\rangle$, the effective distance $r_{\text {eff,3 }}$ is still somewhat lower than the true mean because of the bias introduced by the $1 / r_{12}^{3}$ weighting in Eq. (9). Second-order polynomial fits to the dependences shown in Fig. 5 give the following empirical calibrations of the true mean value in terms of the effective values obtained experimentally from the average splitting and second moment:

$$
\begin{aligned}
\frac{\left\langle r_{12}\right\rangle}{\sigma_{r_{12}}}= & 1.193 \pm 0.051+(0.789 \pm 0.016) \frac{r_{e f f, 3}}{\sigma_{r_{12}}} \\
& +(0.0113 \pm 0.0012)\left(\frac{r_{e f f, 3}}{\sigma_{r_{12}}}\right)^{2}
\end{aligned}
$$

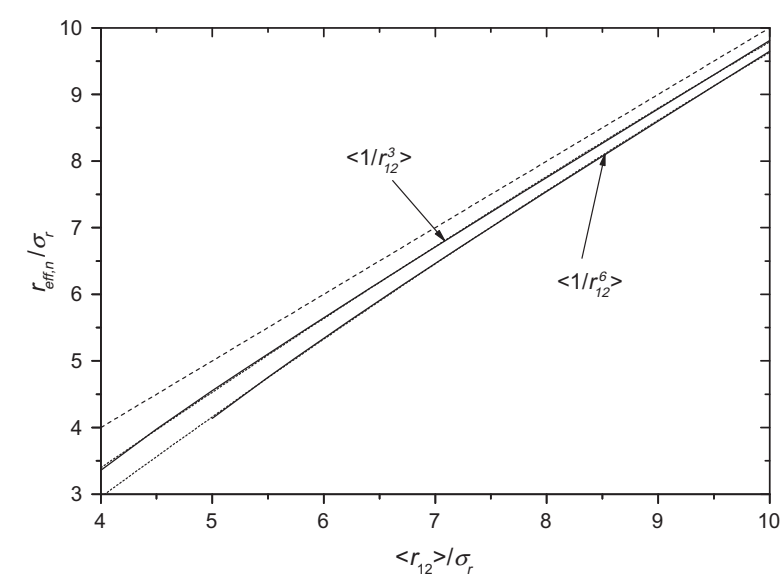

Fig. 5. Effective vs. mean interspin distance. Dependences of the effective mean separation, defined by: $r_{\text {eff. } 3}=\left\langle 1 / r_{12}^{3}\right\rangle^{-1 / 3}$ and $r_{\text {eff. } 6}=\left\langle 1 / r_{12}^{6}\right\rangle^{-1 / 6}$, on the true mean value (solid lines), where the dashed line is: $r_{e f f, n}=\left\langle r_{12}\right\rangle$. The dotted lines are secondorder polynomial fits.

$$
\begin{aligned}
\frac{\left\langle r_{12}\right\rangle}{\sigma_{r_{12}}}= & 1.759 \pm 0.052+(0.723 \pm 0.016) \frac{r_{\text {eff }, 6}}{\sigma_{r_{12}}} \\
& +(0.0138 \pm 0.0011)\left(\frac{r_{e f f, 6}}{\sigma_{r_{12}}}\right)^{2}
\end{aligned}
$$

where the range of fitting is $\left\langle r_{12}\right\rangle / \sigma_{r_{12}}=4-10$ for $n=3$ (i.e., Eq. (10)), and $\left\langle r_{12}\right\rangle / \sigma_{r_{12}}=5-10$ for $n=6$ (i.e., Eq. (11)).

\section{Data for an $\alpha$-helical peptide}

Mean inter-spin distances and distribution widths have been determined by convolution and deconvolution methods for $\alpha$-helical peptides ( $\left.\mathrm{Ala}_{4} \mathrm{Lys}\right)_{4}$ Ala with methane thiosulphonate spin labels attached at two differently spaced cysteine residues substituted in the sequence [10]. These peptides are denoted $4 \mathrm{~K}\left(n_{1}, n_{2}\right)$ where $n_{1}$ and $n_{2}$ are the positions of the residues labeled. For an $\alpha$-helix, the distance between residues is determined uniquely by $\Delta n=n_{2}-n_{1}$. Table 1 gives the values of $\left\langle r_{12}\right\rangle$ and $\sigma_{r_{12}}$ obtained from convolution/deconvolution for different values of $\Delta n$, assuming unlike spins in each case. Additionally, effective inter-spin distances have been determined from average dipolar splittings and from spectral second moments, for the same series of peptides. These values of

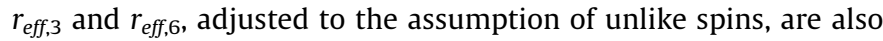
given in Table 1 . As expected, the effective distances are consistently smaller than the mean values. Correction of the effective values by using Eqs. (10) and (11), respectively, results in distances that more closely approach the mean values, for a fixed distribution width given by $\sigma_{r_{12}}=0.38 \mathrm{~nm}$. Only the second moment result for $\Delta n=4$, the shortest distance, differs appreciably from the mean value. Second moment calculations are particularly sensitive to noise and also to truncation in the wings of the spectra, which correspond to the smallest distances.

Previously, structural consistency of the results from average splittings of deconvoluted spectra was demonstrated with a geometrical model in which the nitroxide was fixed at constant radial distance from the helix axis [6]. A distance of $0.67 \mathrm{~nm}$ was found to give best agreement with the experimental splittings for a $4 \mathrm{~K}\left(n_{1}\right.$, $n_{2}$ ) series. However, molecular modeling of these peptides implies larger distances [10]: a radial distance of $0.97 \mathrm{~nm}$ would best fit the mean distances obtained from molecular modeling. This further supports the expectation that effective distances derived from absolute first moments lie below the mean.

The size of the corrections given in Table 1 are of the order of $+0.27 \mathrm{~nm}$ for $r_{e f f, 3}$ and $+0.39 \mathrm{~nm}$ for $r_{\text {eff, },}$. For comparison, the random experimental errors for $r_{e f f, 6}$, obtained from the second moment, range from $\pm 0.05 \mathrm{~nm}$ for the smallest $r_{\text {eff, } 6}$ to $\pm 0.73 \mathrm{~nm}$ for the largest $r_{\text {eff, } 6}$ [10]. Thus, depending on the distance range, corrections can be considerably larger than, comparable to, or smaller than the random error. In all cases, however, they are a systematic error that should be corrected.

\begin{tabular}{|c|c|c|c|c|c|}
\hline$\Delta n$ & $\left\langle r_{12}\right\rangle\left(\sigma_{r_{12}}\right)(\mathrm{nm})^{\mathrm{a}}$ & $\left\langle 1 / r_{12}^{3}\right\rangle^{-1 / 3}(\mathrm{~nm})^{\mathrm{b}}$ & $r_{c o r r, 3}(\mathrm{~nm})$ & $\left\langle 1 / r_{12}^{6}\right\rangle^{-1 / 6}(\mathrm{~nm})^{\mathrm{c}}$ & $r_{\text {corr, } 6}(\mathrm{~nm})$ \\
\hline 4 & $1.17(0.38)$ & 0.80 & 1.11 & 0.91 & 1.36 \\
\hline 7 & $1.31(0.40)$ & 0.89 & 1.18 & 0.97 & 1.40 \\
\hline 8 & $1.56(0.38)$ & & & 1.20 & 1.59 \\
\hline 9 & $1.82(0.37)$ & 1.58 & 1.78 & 1.55 & 1.88 \\
\hline 11 & $1.70(0.38)$ & & & 1.32 & 1.69 \\
\hline
\end{tabular}

Table 1

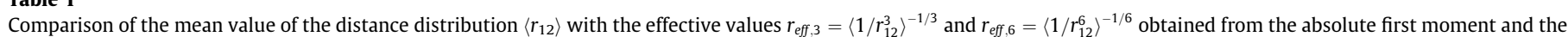
second moment, respectively, for $\alpha$-helical $4 \mathrm{~K}\left(n_{1}, n_{2}\right)$ peptides spin-labelled on residues $\Delta n=n_{2}-n_{1}$ apart.

\footnotetext{
a Mean and standard deviation of $r_{12}$ : average values determined by convolution and deconvolution assuming unlike spins. Data from Ref. [10].

b Effective mean value of $r_{12}$ obtained from absolute first moment after deconvolution, i.e., $r_{\text {eff,3. }}$. Data from Ref. [6] corrected to give values for unlike spins. $r_{\text {corr, } 3}$ is the value of $r_{\text {eff, } 3}$ corrected according to Eq. (10), with $\sigma_{r_{12}}=0.38 \mathrm{~nm}$.

c Effective mean value of $r_{12}$ obtained from second moment after integration to give the absorption spectrum, i.e., $r_{\text {eff.6. }}$ Data from ref. [10] corrected to values for unlike spins. $r_{\text {corr, } 6}$ is the value of $r_{\text {eff, } 6}$ corrected according to Eq. (11), with $\sigma_{r_{12}}=0.38 \mathrm{~nm}$.
} 


\section{Conclusion}

These results emphasize that one expects effective distances deduced both from the second moment of the absorption spectrum, and from the mean splitting in a dipolar deconvolution, to be consistently smaller than the true mean distance. Corrections to the effective values can be made by assuming a Gaussian distribution, if one has a reasonable estimate of the standard deviation. In principle, one could combine measurements of average splitting (i.e., absolute first moment) and second moment to give estimates of both $\left\langle r_{12}\right\rangle$ and $\sigma_{r_{12}}$ by combining Eqs. (10) and (11). But it is important to do this with the same spectral dataset. It is also possible that the present approach might be applied to analyse halffield resonances in dipolar-coupled spin systems.

\section{Acknowledgment}

I thank Christian Griesinger and the Department of NMR-based Structural Biology for financial assistance.

\section{References}

[1] L.J. Berliner, G.R. Eaton, S.S. Eaton, Distance Measurements in Biological Systems by EPR, Kluwer Academic Publishers, New York, 2000.

[2] H.J. Steinhoff, Inter- and intra-molecular distances determined by EPR spectroscopy and site-directed spin labeling reveal protein-protein and protein-oligonucleotide interaction, Biol. Chem. 385 (2004) 913-920.

[3] G. Jeschke, Determination of the nanostructure of polymer materials by electron paramagnetic resonance spectroscopy, Macromol. Rapid Commun. 23 (2002) 227-246.
[4] P.G. Fajer, L. Brown, L. Song, Practical pulsed dipolar ESR (DEER), in: M.A. Hemminga, L.J. Berliner (Eds.), EPR Spectroscopy in Biological Systems by EPR, Springer Science+Business Media, New York, 2007, pp. 95-128.

[5] H.J. Steinhoff, O. Dombrowsky, C. Karim, C. Schneiderhahn, Two dimensional diffusion of small molecules on protein surfaces: an EPR study of the restricted translational diffusion of protein-bound spin labels, Eur. Biophys. J. 20 (1991) 293-303.

[6] M.D. Rabenstein, Y.-K. Shin, Determination of the distance between two spin labels attached to a macromolecule, Proc. Natl. Acad. Sci. U. S. A. 92 (1995) 8239-8243.

[7] H.J. Steinhoff, N. Radzwill, W. Thevis, V. Lenz, D. Brandenburg, A. Antson, G. Dodson, A. Wollmer, Determination of interspin distances between spin labels attached to insulin: comparison of electron paramagnetic resonance data with the X-ray structure, Biophys. J. 73 (1997) 3287-3298.

[8] C. Altenbach, K.J. Oh, R.J. Trabanino, K. Hideg, W.L. Hubbell, Estimation of interresidue distances in spin labeled proteins at physiological temperatures: experimental strategies and practical limitations, Biochemistry 40 (2001) $15471-15482$.

[9] W. Xiao, Y.-K. Shin, EPR spectroscopic ruler, the deconvolution method and its applications, in: L.J. Berliner, G.R. Eaton, S.S. Eaton (Eds.), Distance Measurements in Biological Systems by EPR, Kluwer Academic Publishers, New York, 2000, pp. 249-276.

[10] J.E. Banham, C.M. Baker, S. Ceola, I.J. Day, G.H. Grant, E.J.J. Groenen, C.T Rodgers, G. Jeschke, C.R. Timmel, Distance measurements in the borderline region of applicability of CW EPR and DEER: a model study on a homologous series of spin-labelled peptides, J. Magn. Reson. 191 (2008) 202-218.

[11] N. Radzwil, K. Gerwert, H.J. Steinhoff, Time-resolved detection of transient movement of helices $\mathrm{F}$ and $\mathrm{G}$ in doubly spin-labeled bacteriorhodopsin, Biophys. J. 80 (2001) 2856-2866.

[12] J.H. Van Vleck, The dipolar broadening of magnetic resonance lines in crystals, Phys. Rev. 74 (1948) 1168-1183.

[13] E.J. Hustedt, A.H. Beth, Nitroxide spin-spin interactions: applications to protein structure and dynamics, Ann. Rev. Biophys. Biomol. Struct. 28 (1999) 129-153.

[14] G.E. Pake, Nuclear resonance absorption in hydrated crystals: fine structure of the proton line, J. Chem. Phys. 16 (1948) 327-336.

[15] J.S. Hyde, J.R. Pilbrow, A moment method for determining isotropic g-values from powder electron paramagnetic resonance, J. Magn. Reson. 41 (1980) 447 457. 